\title{
Using Center-Specific Medicare Data to Compare Cancer Care Outcomes: Are We Seeing the Whole Playing Field or Just a Blade of Grass
}

\author{
Thomas A. Aloia, MD, MHCM, FACS ${ }^{1}$ \\ Department of Surgical Oncology, University of Texas MD Anderson Cancer Center, Houston, TX
}

In this issue of Annals of Surgical Oncology, the Ohio State University outcomes research group has published "The Impact of Dedicated Cancer Centers on Outcomes Among Medicare Beneficiaries Undergoing Liver and Pancreatic Cancer Surgery." ${ }^{1}$ The report describes a rigorous statistical methodology demonstrating that hepatopancreatobiliary (HPB) surgical outcomes for Medicare beneficiaries who underwent surgery between 2013 and 2015 at Prospective Payment System (PPS)-exempt cancer centers experienced fewer short-term postoperative complications than those who had surgery at non-PPS exempt hospitals.

Because Alliance of Dedicated Cancer Center (ADCC) hospitals are generally higher-volume centers for complex surgical oncology, particularly for operations that treat HPB malignancy, from a surgical perspective, the observed patient outcomes differences could be attributed to simple volume-outcome relationships. In addition, these outcomes differences may reflect recognized and unrecognized variability in patient risk including socioeconomic profiles, comorbidities, psychosocial distress, access to care, and other related factors. Alternatively, they may reflect a higher level of technical ability, teamwork, ancillary support, nursing skill, and multidisciplinary coordination at the PPS-exempt centers. Likely, the answer is a multifactorial combination of all these factors.

(C) Society of Surgical Oncology 2019

First Received: 25 July 2019;

Published Online: 16 August 2019

T. A. Aloia, MD, MHCM, FACS

e-mail: taaloia@mdanderson.org
However, the most interesting part of this publication actually is in the Methods section, not the Results section. This report is at the cutting edge of a recent methodologic shift in Medicare Inpatient Standard Analytic Files database-related research. With the newly upgraded access for health care delivery research groups to identify each care-providing hospital individually in the data sets comes the ability to compare performance between hospitals, hospital networks, and other groupers including ADCC, NCI-designated, and countless others.

But with this added ability comes added responsibility. Editors and authors need to demonstrate discipline and rigor in their disclosures and discussions of data set limitations, as Mehta et al. ${ }^{1}$ have done in this report. These data sets speak to a limited sliver of outcomes that focus on short-term physician-centric harm metrics. They contain no data on cancer stage, patient experience, patient-reported quality of life, functional degree of recovery, or return to intended oncologic therapy, ${ }^{2}$ much less on cancer-specific long-term survivals. Likewise, these data do not speak to coordination of care and/or multidisciplinary oncology care, which stand at the center of population-level improvements in cancer outcomes.

As such, in the same way that we could never use these limited data sets to justify the continued existence of the PPS exemption for the ADCCs, we should not weaponize these data to argue that the exemption should be stripped away, as was recently done. ${ }^{3}$ The Medicare data set is simply not the right scale for that measurement. The outcomes for a relatively small number of older cancer patients with a unique combination of stage, performance status, access, and favorable biology that afforded them the opportunity for a complex operation almost a decade ago 
cannot and should not be used to set (or even comment on) health policy regarding future payment models for global cancer care in the United States.

The aforementioned data may be used to inform ongoing discussions regarding regionalization of complex cancer care. The argument that in an ideal world, every pancreatic cancer patient should have his or her Whipple with a highvolume surgeon at a high-volume and multidisciplinarystaffed center is as much a given as it is an impossibility. Although strides have been made toward this goal, no mechanism exists for full operationalization of surgical regionalization, and patients are increasingly choosing convenience over outcomes in determining their selection of treatment location.

Given these realities, analyses that indicate superior patient outcomes at ADCCs are unlikely to increase the gravitational pull of patients to main-campus ADCC hospitals. Instead, these data can be used to encourage ADCCs to expand access to better cancer surgery outcomes by building quality-controlled regional networks that carry their "secret sauce" to communities at a distance from their main-campus location, and by educating non-affiliated centers on the latest breakthroughs in treatment methods and care models.

As we call on ADCCs to do this, we must understand that it is impossible to replicate every aspect of maincampus care at regional sites. Lower-volume generalists with fewer ancillary resources than centers of excellence will always need to play a prominent role in cancer care. Therefore, the patient outcomes may never be equivalent inside or outside of an ADCC's regional affiliated network, and research questions that pose equivalence as the hypothesis will always find disappointing results. Likewise, publications that use the Medicare data set to impugn the concept of cancer center networks without commenting on the substantial negative outcomes delta between the regional affiliate and the next available non-affiliated option do not move the ball forward either. ${ }^{4}$ The primary promise of the PPS exemption and its rationale for existence is not to equilibrate outcomes across the United States, but to improve cancer care continually through rapid dissemination of research breakthroughs, innovative care models, and education.

Because high-quality, whole-population cancer care is a sufficiently challenging race without a finish, there is no reason to overextend the capacity of limited data sets to place additional obstacles in front of the runners.

\section{FUNDING None.}

DISCLOSURE There are no conflicts of interest.

\section{REFERENCES}

1. Mehta R, Ejaz A, Hyer J, Tsilimigras D, White S, Merath K, et al. The impact of dedicated cancer centers on outcomes among medicare beneficiaries undergoing liver and pancreatic cancer surgery. Ann Surg Oncol. 2019. https://doi.org/10.1245/s10434-0 19-07677-1.

2. Aloia TA, Zimmitti G, Conrad C, Gottumukalla V, Kopetz S, Vauthey JN. Return to intended oncologic treatment (RIOT): a novel metric for evaluating the quality of oncosurgical therapy for malignancy. J Surg Oncol. 2014;110:107-14.

3. Merkow RP, Yang AD, Pavey E, Song MW, Chung JW, Bentrem DJ, et al. Comparison of hospitals affiliated with PPS-exempt cancer centers, other hospitals affiliated with NCI-designated cancer centers, and other hospitals that provide cancer care. JAMA Intern Med. 2019. https://doi.org/10.1001/jamainternmed.2019. 0914.

4. Hoag JR, Resio BJ, Monsalve AF, Chiu AS, Brown LB, Herrin J, et al. Differential safety between top-ranked cancer hospitals and their affiliates for complex cancer surgery. JAMA Netw Open. 2019;2:e191912.

Publisher's Note Springer Nature remains neutral with regard to jurisdictional claims in published maps and institutional affiliations. 